\title{
Revistas periféricas de generación interna en la Universidad Autónoma de Chihuahua, México: identificación de indicadores para la calidad
}

\author{
Javier TARANGO \\ Universidad Autónoma de Chihuahua. Facultad de Filosofía y Letras \\ jtarango@uach.mx \\ Arturo Iván RUIZ-DomíNGUEZ \\ Universidad Autónoma de Chihuahua. Licenciatura en Ciencias de la Información \\ air_ruiz2@msn.com
}

Recibido: Julio 2012

Aceptado: Septiembre 2012

Resumen: Este artículo representa el resultado de una investigación en relación a siete publicaciones periódicas editadas por la Universidad Autónoma de Chihuahua (México), las cuales constituyen su catálogo total, para lo cual se procedió a desarrollar un análisis bibliométrico que identificara las principales regularidades y así determinar las condiciones de calidad editorial que actualmente observan para la posterior derivación de propuestas hacia el cambio. Tales publicaciones representan un mecanismo para la divulgación del conocimiento a través del trabajo individual y colectivo de sus académicos, mismas que fueron calificadas como publicaciones periódicas de carácter periférico y no de corriente principal (mainstream), debido a una serie de limitaciones en sus procesos de identificación de indicadores de impacto y visibilidad. De acuerdo a los resultados obtenidos se derivan diversas recomendaciones hacia la calidad y posible cambio de estatus editorial, tales como: constancia y mejora en los procesos de recepción y evaluación de los artículos a publicar, definición de comités científicos integrados por miembros de reconocido prestigio, identificación de políticas editoriales estrictas hacia la estandarización, aumento en la visibilidad de las publicaciones y acotamiento temático evitando la dispersión disciplinar sin especialización.

Palabras clave: Revistas periféricas; Revistas de corriente principal; Estudios bibliométricos; Revistas de divulgación interna; Universidades mexicanas.

\section{Peripheral journals from own generation at the Autonomous University of Chihuahua, México: identification of indicators for quality}

\begin{abstract}
This paper represents the results of an investigation into seven serials edited by the Autonomous University of Chihuahua (Mexico), which constitute its full catalog, for which we proceeded to develop a bibliometric analysis to identify the main regularities and determine editorial quality conditions currently observed for the subsequent derivation of proposals for change. These publications represent a mechanism for the dissemination of knowledge through individual and collective work of its scholars, which were classified as peripheral journals and
\end{abstract}


not mainstream, due to a number of limitations in their processes identifying indicators of impact and visibility. According to the results derived several recommendations to quality and possible change of status editorial, such as consistency and improvement in the processes of reception and evaluation of papers to publish, definition of scientific committee composed of members of recognized standing, identifying strict editorial policies towards standardization, increased visibility of publications and Theme shoulder avoiding dispersion unskilled discipline without specialization.

Keywords: Peripheral journals; Mainstream journals, Bibliometric studies; internal generation journals; Mexican universities.

\section{INTRODUCCIÓN}

Las ciencias de la información como disciplina científica observa como característica peculiar ser interdisciplinaria, ya que interpreta varias ciencias e incluso se autoevalúa buscando su propia determinación de parámetros, por lo cual, aplica el método científico y se relaciona con las disciplinas básicas (archivología, bibliografología y bibliotecología), por tanto, el abordaje que hace en la evaluación de las ciencias la posicionan como una disciplina comunicativoinformativa (Satién Quesada y Gorbea Portal, 1994).

Actualmente, los campos de acción profesional de las ciencias de la información se extienden a otras dimensiones, en este caso, a las cuestiones relacionadas con la evaluación de las regularidades en la información científica, por tanto, se demandan conocimientos firmes de bibliografía, historia y metodología de la investigación como elementos rectores para la definición de la cualidad de los productos, manifestados estos a través de publicaciones, tanto internas de las propias organizaciones o a través de la búsqueda de los docentes e investigadores de distintas alternativas ajenas a sus entornos laborales (López Ornelas, 2004).

La producción científica forma parte de un proceso complejo, con actores sociales variados que no se pueden encasillar en el concepto de los personajes científicos, ya que en la actualidad, su actividad merece una especial atención y un reconocimiento a los procesos de producción y distribución del conocimiento y su efecto social (Márquez Nerey, 2010). Los medios a través de los cuales se difunden los hallazgos de conocimiento merecen también una especial atención, ya que de ellos depende que la producción científica se convierta en comunicación científica formal.

Se debe partir del hecho de que las universidades están obligadas a ir más allá de la mera docencia como mecanismo de transferencia de conocimiento y complementar su función con base a otras actividades necesarias relacionadas con la investigación y los procesos de divulgación o difusión del conocimiento (Sánchez Hernández, Delgado Sánchez y Soria Ramírez, 2007). De hecho, las actividades de docencia pueden estar vinculadas directamente con el uso de 
información y las demás actividades mencionadas, con la generación de conocimiento manifestado este a través de productos de investigación científica, publicados a través de diversos medios formales de divulgación científica.

Las universidades buscan medios de producción científica a través de sus docentes e investigadores, pretendiendo además, convertirse en mecanismos de divulgación de la ciencia por medio de sus propias publicaciones. Cuando se evalúa la producción interna de las instituciones de educación superior se permite identificar de ellas: la selección de documentos que el autor incluyó en sus referencias, identificación de las características temáticas de la literatura, evaluación bibliográfica y de colecciones, registro de la historia de la ciencia, determinación de revistas núcleo, así como determinación de élites intelectuales y modelos de distribución de contenidos, entre otros (Dávila Rodríguez y otros, 2009).

El comportamiento de las publicaciones científicas debe analizarse de forma sistemática. El primer análisis permite saber el número total o acumulado de artículos y de las citas a las que éstas se han hecho acreedores desde el año de su publicación. Sin embargo, sucede con frecuencia que no se pueda determinar la importancia o impacto de un artículo en relación con las citas recibidas en un periodo determinado, ya que éste no ha sido difundido suficientemente y su influencia no se ha logrado capitalizar, ya que los medios de divulgación no alcanzan a ser analizadas desde la perspectiva de su factor de impacto, además de que cabe la posibilidad de que no se logre un cambio de estatus, manteniéndose como publicaciones periféricas de baja o mediana calidad.

Pero eso no es todo, se requiere además la divulgación en otras formas, entre las cuales están los medios internos de las universidades, generalmente enfocadas en el nivel de revistas periféricas (que no significa que observen niveles de baja calidad editorial), la cuestión radica en la posibilidad primero, de convertir todo lo que de las instituciones de educación superior emane y segundo, se convierta en comunicación científica, la cual es definida por Gutiérrez Couto, Blanco Pérez y Casal Acción (2004), como el proceso de distribución y recepción de la información científica en la sociedad, considerada como un sinónimo de comunicación académica, siempre y cuanto suceda a través de canales formales, ya que es la manera efectiva de llevar a cabo la divulgación científica.

\section{IDENTIFICACIÓN DE LAS FUENTES DE DIVULGACIÓN}

Más allá de los medios de divulgación que apoyan la distribución de las revistas, se hace necesario entender la definición y clasificación que estas observan dentro de una concepción actual, considerando que dentro de cualquier nivel de publicación, serán consideradas como revistas científicas. UNESCO (2003) define a las revistas científicas como la publicación periódica que presenta especialmente artículos científicos, escritos por autores diferentes e incluyen en 
sus contenidos información de actualidad sobre investigación y desarrollo de cualquier área de la ciencia.

Otras características, que según Jiménez Sánchez y Castañeda Hernández (2003) que observan las revistas científicas es que tienen un nombre distintivo, se publican a intervalos regulares (por lo general varias veces al año) y cada edición está numerada o fechada consecutivamente. Su componente básico, el artículo científico, es un escrito en prosa, de regular extensión, publicado como una contribución al progreso de una ciencia.

De acuerdo a la American Library Association (ALA, 2009), la revista científica es un medio de divulgación que resalta dentro de sus contenidos la presencia de artículos son originales, sobre investigación y desarrollo en un campo determinado de la ciencia, lo que indica ser el medio elegido por los investigadores para difundir el primer escrito de sus resultados, por lo que no se tiene una versión escrita anterior y su contenido es una contribución al conocimiento.

Mendoza y Paravic (2006), así como Samar y Ávila (2004), consideran que la base de las revistas científicas está sustentada en el artículo científico, el cual es definido como un informe escrito donde se representan los resultados originales de una investigación y se convierte en publicación válida o publicación científica primaria, cuando se publica por primera vez y su contenido informa lo suficiente para que se puedan evaluar las observaciones, repetir los experimentos y evaluar los procesos intelectuales realizados por los autores involucrados en la creación del documento.

\subsection{CLASIFICACIÓN DE LAS PUBLICACIONES PERIÓDICAS}

Para dimensionar el nivel en que se pueden encontrar las publicaciones desarrolladas por las universidades para divulgar los hallazgos sobresalientes generados por sus docentes e investigadores, se hace necesario entender la clasificación de las revistas de acuerdo a la propuesta integrada por López Ornelas y Cordero Arroyo (2005), quienes parten de desarrollar una taxonomía de acuerdo a las entidades que las editan y son representadas en tres tipos:

a) De primer nivel, cuando son editadas y publicadas por sociedades científicas reconocidas internacionalmente, de costos poco elevados pues cuentan con recursos económicos provenientes de los asociados.

b) De segundo nivel, cuando los procesos de edición, publicación y comercialización se realizan a través de grandes compañías transnacionales, lo que además les confiere prestigio, pero tienen el inconveniente de tener un elevado costo de suscripción y con ello se limita su circulación.

c) De tercer nivel, editadas y publicadas por entidades públicas (universidades, hospitales, centros de investigación, etc.), instituciones que las imprimen 
con problemas propios de la dependencia administrativa en donde se general (los cuales pueden ir desde bajos presupuestos de operación, cambios de funcionarios, baja divulgación, etc.), generalmente caracterizadas por irregularidades en los períodos de publicación, distribución y difusión.

Si bien la clasificación anterior se refiere al origen de la publicación, otro aspecto importante en la tipificación de recursos periódicos es de acuerdo a los tipos de contribuciones que estos reciben:

a) De información, en donde se divulgan programas (científicos, técnicos, educativos, etc.), reuniones de interés científico o artículos breves que informan sobre personas destacadas en el área de la ciencia y el arte.

b) Primarias, en donde se publican artículos científicos originales.

c) Secundarias (de resúmenes), en donde se compila el contenido de revistas primarias.

d) Terciarias (revistas de progresos científicos o tecnológicos), en donde se publican informes resumidos de programas de investigación.

Una tercera clasificación se refiere a la diferenciación de acuerdo al público al que están dirigidas, en donde las revistas son identificadas en la siguiente tipología:

a) Boletines (gacetas o newsletters), en donde se difunden noticias o información de interés práctico (eventos o convocatorias).

b) De divulgación (conocidas como magazines), dirigidas a público general para informar de temas científicos, culturales, artísticos, etc., en lenguaje sencillo.

c) Académicas (Scientific journals), dirigidas a un grupo que se dedica a estudiar ciertos temas (especialistas), que cumplen con exigencias de calidad editorial y que estudian ciertos temas de carácter disciplinario en donde se expresan resultados de un estudio realizado.

El International Council for Science (1999), considera que los criterios de clasificación de las revistas antes presentados requieren agregarse el criterio relacionado con la cobertura de suscriptores, por lo que las publicaciones académicas se agruparían en dos tipos:

a) Revistas pequeñas, que se caracterizan por ser revistas de reciente creación, con pocos suscriptores, escasa difusión, editadas por instituciones educativas, sin aval de alguna sociedad científica o empresa editorial y no están consideradas en los índices en organismos internacionales que 
regulan la calidad de la investigación, ciencia y tecnología, tales como: Colciencias de Colombia, CONICYT de Chile, CONACYT de México, e incluso de forma global en el Institute for Scientific Information (ISI).

b) Revistas grandes, las cuales se caracterizan por ser difundidas a grandes poblaciones de lectores, publican artículos originales, son editadas tanto por instituciones de investigación, educación, sociedades científicas $\mathrm{u}$ organizaciones editoriales de reconocido renombre. Pueden ser impresas o electrónicas, ambas de forma simultánea o primero en un formato y luego en el otro.

\subsection{PERSPECTIVAS DE LAS REVISTAS PERIFÉRICAS}

La no pertenencia de las revistas a diversos organismos de evaluación de la calidad de las publicaciones, tales como el ISI o los organismos de evaluación nacional de cada país en particular, no presupone que al entrar en la categoría de publicaciones periféricas no sean medios de divulgación de las ciencia o que necesariamente pertenezcan a los modelos de divulgación de países económica y socialmente marginados y por tanto, ofrezcan un acervo de conocimiento atrasado. Debe reconocerse que no todas las revistas periféricas pueden ser catalogadas como de calidad.

Gorbea Portal y Suárez-Balseiro (2007) definen a las revistas periféricas como aquel grupo de publicaciones que no aparecen procesadas en los grandes sistemas de difusión de la ciencia, por tanto, y de acuerdo a los parámetros aceptables de publicaciones de corriente principal o mainstream, como el Social Citation Index (SCI) o el Science Citation Index (SCI) que representa una fuente universal de amplia divulgación y criterios de aceptación. Todas la publicaciones ofrecen un arsenal de datos de citaciones para definir la forma como se usa el conocimiento científico a través de las publicaciones, pero mientras la publicación no esté inmersa en un índice reconocido, no será posible evidencias comparaciones contra el área, la región o el país, así como identificar diversos indicadores de la producción del conocimiento (López-López, 2009).

Autores como Núñez Jover (2006) defienden la posibilidad de abrir un camino de valoración a aquellas prácticas científicas consideradas como ciencia periférica y por tanto, que tienen medios específicos de divulgación en revistas periféricas y naturalmente, que no están incluidas dentro del mainstream o corriente principal. La asociación de estas publicaciones con países subdesarrollados y además la baja producción de modesta contribución a nivel mundial imposibilitan la tendencia hacia el reconocimiento y sobre todo al cambio de paradigma en todos los sentidos.

Como se menciona anteriormente, la defensa de las publicaciones periféricas es constante, aunque sus resultados de participación en las contribuciones a la ciencia no ayudan a una determinación hacia el reconocimiento. Es por eso que tradicionalmente la ciencia se centra en los países desarrollados, quienes además 
controlan y vigilan la integración de mecanismos formales de las publicaciones (propias y ajenas) convirtiendo a la producción científica en una industria con capacidad de divulgación efectiva de la ciencia, con contribuciones al desarrollo de la docencia y la firme conciencia de la importancia de creación y aplicación de políticas científicas hacia la producción y comunicación científica.

La conveniencia de abordar los procesos de evaluación de revistas científicas periféricas estriba en que estas representan un esfuerzo de las instituciones por divulgar los productos desarrollados por sus docentes e investigadores quienes publican, de forma aceptable, en revistas locales, ya que la mayoría de las veces son en el idioma propio. Ríos Ortega (2006) prestó especial importancia al estudio de las revistas científicas periféricas ya que las considera como fundamentales en el desarrollo de la ciencia regional, además de la pretensión de cada publicación de esta naturaleza por pasar al grupo de revistas científicas., enfrentando para ello dificultades, tales como la continuidad, el arbitraje y el acceso a propuestas de artículos a publicar.

Existe una determinación de los docentes e investigadores en universidades a mantener su vigencia en el desarrollo de publicaciones en las revistas integradas dentro del grupo de corriente principal o mainstream, sin embargo, existen autores que se inclinan por fomentar el desarrollo de sus colaboraciones en revistas periféricas, argumentando que estas permiten:

a) Mantener el acceso y distribución a las instituciones con las revistas científicas que producen.

b) Contemplar canales y mecanismos de distribución de las comunicaciones científicas, ya sea de forma impresa o usando la tecnología.

c) Considerar al Open Access como un como medio de distribución del conocimiento científico.

Las vías antes mencionadas deberán ser consideradas por las instituciones de educación superior que generan revistas como medio de favorecer la publicación de artículos científicos por sus propios miembros, cuya divulgación sea más amplia y efectiva. La determinación de elementos clave para evaluar una revista, entre los que se pueden mencionar: frecuencia de la publicación, país de origen, idioma, temáticas abordadas, estatus de la publicación (activa o cerrada), fechas de inicio y terminación, tipo de publicación (académicas/científicas, noticieros, comercio, directorio, series monográficas, boletines, gobierno, bibliografía, otras), son elementos recomendados por Gorbea Portal y Ávila Uriza (2009), con el fin de identificar el estado actual de las publicaciones evaluadas.

La imagen de una institución de educación superior o de investigación estará sustentada en la concepción que se tenga de su producción científica, a la determinación que se tenga de establecer mecanismos de comunicación científica y el nivel al que se pretende llegar, así como a los niveles de producción de sus 
miembros, así como a la potencialidad para ello y disposición hacia el cambio de paradigma entre su realidad y su potencialidad en su contribución al desarrollo de la ciencia. La revista periférica se convierte en el mecanismo ideal para tales fines.

\section{JUSTIFICACIÓN DEL PROBLEMA}

Las universidades como entes generadoras de conocimiento establecen diversos mecanismos de divulgación de sus hallazgos, los cuales se pueden agrupar en dos grandes rubros: 1). Las publicaciones externas a la misma organización (las cuales son la mayoría), y 2) aquellos mecanismos de divulgación interna (que regularmente son pocos y su comportamiento va de acuerdo con las condiciones particulares de cada institución que las genera).

Para el caso de este estudio, se observó que las publicaciones que generan las universidades mexicanas corresponden al rubro de periféricas (por lo que no son evaluadas por su factor de impacto), suelen presentar períodos de difusión con características irregulares y están favorecidas en la selección de propuestas de publicación provenientes de sus propios docentes e investigadores.

En vista de estos hechos, algunas de estas publicaciones tienen inconvenientes en definición de estándares de diversos aspectos de evaluación y presentación de contenidos, suelen estar en procesos de obtención de números normalizados (ISSN, DOI, etc.) y no cuentan con comités editoriales estrictos que regulen la publicación. Por tanto, el presente estudio se justifica en la necesidad de diagnosticar la condición real y precisa de las publicaciones internas, tomando como referencia el estudio del caso de una universidad en particular, a través de una descripción general del estado de tales publicaciones en diferentes períodos, determinando así su condición editorial y las posibilidades de cambio en su estatus a partir de la inclusión de indicadores de calidad, a fin de favorecer mayor impacto en el registro de evaluaciones institucionales en relación con la producción y comunicación científica.

\section{DELIMITACIÓN DEL ESTUDIO}

Este trabajo de investigación corresponde a un estudio bibliométrico sobre publicaciones periódicas promovidas por la Universidad Autónoma de Chihuahua (UACH), México, como parte de sus mecanismos de divulgación del conocimiento generado dentro de su misma estructura, a través de la colaboración de sus propios docentes e investigadores.

El presente estudio aplica a siete publicaciones periódicas de las distintas entidades organizacionales de la UACH que las generan, tanto en modalidades impresa y electrónica, según sea el caso de la publicación. Dadas las condiciones de las publicaciones, la evaluación se desarrolla en el trascurso de los últimos años de la edición de las mismas en donde se haya observado constancia y continuidad, 
ya que en algunas publicaciones su última época es representada por una publicación continua de 15 años.

Además, para el caso de otras publicaciones con mayor período de publicación, se cuentan con hasta 50 años en edición, pero con el inconveniente de no haberse identificado copia física o electrónica de los artículos contenidos, por lo que los sistemas de medición sólo se aplican según la característica o regularidad de los ejemplares obtenidos al inicio del mes de septiembre del año 2011 cuando se inicia la captura de datos.

Tal como se menciona con anterioridad, el universo de trabajo en cuanto a revistas periféricas identificadas en la $\mathrm{UACH}$ como parte de este estudio fueron siete. Cada una de ellas observa una historia y estructura diferente, además de que cada cual tiene planteados sus propios objetivos y propósitos de crecimiento y desarrollo. A continuación se presenta un bosquejo de cada revista periférica identificada, de acuerdo a la información proporcionada por los responsables de cada publicación:

a) Lecturas Jurídicas. Es una revista publicada por la Facultad de Derecho de la $\mathrm{UACH}$, con periodicidad trimestral y con una edad de 50 años. Actualmente la revista observa un período de edición que corresponde a la sexta época, número 15 del mes de abril de 2012 hasta el momento del inicio de la captura de de los datos de sus artículos considerados para esta investigación. Esta revista actualmente está incluida dentro de LATINDEX.

b) Excelencia Administrativa. Publicada a través de la Facultad de Contaduría y Administración de la UACH a partir de febrero de 2004. Publica trabajos de investigación de sus docentes, así como artículos de interés en ciencias económico-administrativas.

c) REDICEA. Publicada desde 2002 de forma co-editada por la Asociación de Profesores de Contaduría y Administración, A.C. y la Facultad de Contaduría y Administración de la $\mathrm{UACH}$, su propósito es difundir los trabajos realizados por profesores a nivel regional, nacional e internacional en el campo de las ciencias económico-administrativas.

d) Tecnociencia Chihuahua: Revista Arbitrada de Ciencia, Tecnología y Humanidades. Inicia publicación en 2007 por la Dirección de Investigación y Posgrado de la UACH, pertenece a LATINDEX y RedALyC, cuenta con un comité editorial integrado por académicos reconocidos a nivel nacional e internacional considerando la posibilidad de incursionar en procesos de indización.

e) DOXA. Publicación de la Facultad de Ciencias Políticas y Sociales de la UACH con periodicidad semestral a partir del 2005, contando actualmente con cinco números y abordando temas de: sociología, ciencia política, administración pública, relaciones internacionales, ciencias de la comunicación y economía, entre otras. 
f) Metamorfosis. Revista publicada por la Facultad de Filosofía y Letras de la $\mathrm{UACH}$, iniciando en 1968, indicando ofrecer entregas cuatrimestrales son interrupciones notables en la constancia de sus ediciones. Se autodefine como multidisciplinaria ya que aborda temas tales como: lingüística, literatura, filosofía, psicología, educación, arte, comunicación y bibliotecología, a manera de ensayos, artículos, reseñas, cuentos, poemas, traducciones y comentarios.

g) Synthesis. Esta revista data de 1987 (con un receso de 1992 a 1996) y nace bajo el esquema multidisciplinario, siendo la publicación de la UACH con mayor número de ediciones. Pertenece a Latindex.

\section{OBJETIVO GENERAL}

Esta investigación analiza el estado de las publicaciones periódicas editadas por la UACH, mediante la aplicación de sistemas métricos de información, además de describir el estatus actual de las últimas ediciones con regularidad en su publicación en su condición de revistas periféricas, conforme a los datos obtenidos por medio de la captura de la totalidad de artículos para identificar sus indicadores de calidad y determinar la posibilidad de cambio a revistas de corriente principal.

\subsection{OBJETIVOS ESPECÍFICOS}

Los objetivos específicos que pretende lograr este estudio son:

a) Clasificar por área de conocimiento, cada uno de los artículos publicados en las siete revistas generadas por la $\mathrm{UACH}$, a fin de delimitar su agrupación tomando como referencia la clasificación temática-disciplinar propuesta por el Sistema Nacional de Investigadores (SNI) de México.

b) Conformar tablas de descriptivos generales, que incluyan: cantidad de publicaciones, años de publicación, edades máximas y cantidad de autores firmantes, elementos que permitan el desarrollo de un análisis bibliométrico específico.

c) Aplicar los principales sistemas bibliométricos que permitan determinar la condición que observan las publicaciones internas de la UACH en cuanto a su condición y derivar propuestas de elementos de mejora a través de sus indicadores de calidad. 


\subsection{PREGUNTAS DE INVESTIGACIÓN}

Los modelos de medición existentes aplicados a los datos de la captura de artículos, permiten comprobar que:

a) ¿Las condiciones de las publicaciones editadas por la UACH pueden ser consideradas como viables a cambiar de condición a partir de la evaluación de sus indicadores bibliométricos?

b) ¿Los medidores de evaluación bibliométrica se amoldan al tipo de análisis presentado?

c) ¿Los resultados que propician la derivación de las conclusiones llevan a proponer el desarrollo de modificaciones futuras en las publicaciones editadas de la UACH a fin de buscar un cambio en su condición de evaluación de su calidad?

\section{METODOLOGÍA}

Al ser este trabajo una investigación sobre el comportamiento bibliométrico de las revistas de edición interna desarrolladas por la UACH, a las que con anterioridad se han definido como publicaciones periféricas, mismas que están bajo la responsabilidad, tanto de instancias organizacionales centrales (como son las direcciones de extensión y difusión y de las áreas de investigación y posgrado) y algunas entidades organizacionales particulares (en este caso facultades específicas), se siguieron los procedimientos particulares, especialmente usando indicadores relacionados con la obsolescencia, identificación de elites intelectuales, así como de aquellos relacionados con la definición de dispersiónconcentración temática, por mencionar los principales.

El primer paso fue la indagación específica de las revistas de edición interna que correspondieran a las caracterizaciones del estudio, para lo cual se identificaron siete. A partir de la identificación de aquellas publicaciones que comprende el este estudio bibliométrico, se procedió a la recogida de datos relacionados con los artículos incluidos en cada una de las publicaciones y acorde a las necesidades de información demandadas por los indicadores métricos a evaluar, para lo cual fueron considerados únicamente los artículos científicos formalmente publicados por revista, considerando que registran un conjunto de referencias debidamente representadas en el texto, excluyendo únicamente la producción literaria corta, tales como poemas, columnas de opinión, narraciones cortas y reseñas de libros, que se incluyen en algunas de las publicaciones debido a que no es motivo de estudio del presente trabajo.

Dada la complejidad del estudio en relación al número de indicadores métricos a evaluar, el vaciado de los datos en la base principal fue desarrollado en el paquete computacional Excel para captura, análisis y filtrado, ya que era el 
procesador de datos que cubrió la expectativa para el procesamiento de un número amplio de campos, mismos que se interrelacionan para su análisis e interpretación.

La enumeración de los campos de información que se consideraron para integrar el análisis de datos: nombre de la publicación, año de publicación, periodicidad de la publicación, datos de identificación de la publicación (número, mes, etc.), inclusión de comité editorial, nombre del artículo, cantidad de autores, nombre de los autores, área de conocimiento, disciplina científica y lista de referencias de cada artículo publicado.

Respecto al campo de comité editorial, este funciona sólo como indicativo de si contiene o no, ya que de antemano se sabe que al tratarse de publicaciones periféricas, una de las condiciones para clasificar a las publicaciones en esta caracterización es su inclusión de índices, para lo cual se demandaría un consejo editorial para evaluar las publicaciones con probada calidad de sus miembros desde la perspectiva de experiencia, formación disciplinar y prestigio reconocido.

La clasificación de los artículos identificados como universo de investigación fueron clasificados de acuerdo a la disciplina particular de abordaje temático de enfoque, la cual es asignada de forma arbitraria por los autores de esta investigación de acuerdo a lo que indica el título del artículo de forma aproximada, siendo tal asignación considerando las clasificaciones disciplinares del modelo del SNI dentro de sus siete áreas generales de conocimiento que se incluyen posteriormente.

Es importante indicar que la elección del modelo de clasificación disciplinar de las ciencias propuesto por SNI (2012) utilizado para esta investigación, estuvo basado en el hecho de que la UACH es una institución de educación superior, pública, con adscripción actual de 64 investigadores a este organismo, el cual fue creado para reconocer la labor de las personas dedicadas a producir conocimiento científico y tecnológico. Las áreas de trabajo y ciencia en que divide a sus miembros se presentan a continuación:

Área I. Físico-matemáticas y ciencias de la tierra (Astronomía, Ciencias de materiales, Física, Geofísica, Geología, Matemáticas, Oceanografía).

Área II. Biología y química (Biología, Química).

Área III. Medicina y ciencias de la salud (Medicina, Ciencias de la salud, Odontología, Farmacia).

Área IV. Humanidades y ciencias de la conducta (Arquitectura, Antropología, Bellas artes, Biblioteconomía, Educación, Filosofía, Historia, Lingüística, Literatura, Psicología).

Área V. Ciencias sociales (Administración, Ciencia política y administración pública, Comunicación, Contabilidad, Demografía, Derecho y jurisprudencia, Economía, Geografía humana, Sociología y prospectiva).

Área VI. Biotecnología y ciencias agropecuarias (Agronomía, Biotecnología, Medicina veterinaria, Pesca y Zootecnia). 
Área VII. Ingenierías (Aeronáutica, Ambiental, Civil, Comunicaciones, Electrónica y Control, Eléctrica, Computación, Industrial, Materiales, Marina y Portuaria, Mecánica, Minera, Nuclear, Petrolera, Química, Textil.

Para la asignación de área temática y la ciencia a la que pertenece el artículo, estos fueron asignados durante el proceso de la captura, obteniéndose la información del título del artículo cuando resultara fácil de identificar y en el caso que se observara alguna complejidad y con el fin de llegar a una asignación temática correcta y precisa en su definición, se recurrió al análisis breve de los contenidos del documento.

Una vez que la información fue capturada de forma completa, se procedió a separar en columnas nuevas con el indicativo del número de autores según la cantidad de participantes que contiene el grupo firmante, esto con el fin de obtener de forma completa la información de los autores totales, asentándolos para ello por apellidos y nombre, observándose aquí un fenómeno particular, bajo el hecho de que fue necesario generar un catálogo de autoridad con los nombres de los autores, ya que estos, en diversas ocasiones, fueron asentados de forma distinta, usando nombre completos con dos apellidos, un apellido, únicamente primer nombre o dos nombres, por mencionar los casos más típicos.

Las fechas de la referencias fueron obtenidas de los artículos en general de la misma lista de referencia que contienen cada uno de los documentos. Como anteriormente se menciona, en caso de que no se cuente con el artículo fuente o que el artículo no contenga referencias, no se desarrollaron captura de estos datos.

\section{ANÁLISIS DE RESULTADOS}

Este apartado presenta los principales hallazgos encontrados en la evaluación de las fuentes identificadas como revistas periféricas publicadas por la UACH a través de diferentes instancias, mismas que son consideradas como el universo investigativo, a través del cual se desarrolló el estudio bibliométrico. Como se menciona, el estudio comprende los artículos publicados en siete revistas que observan un grado de formalidad y constancia en su publicación con un total de 1,175 documentos identificados, correspondientes al período de 1990 como el más antiguo al 2011 en relación con las publicaciones de más reciente publicación. Debido a la naturaleza no regular de algunas de las publicaciones en sus procesos de edición y su acumulación, carente de un procesamiento bibliotecológico formal, de muchas de ellas no se cuenta con todos los números publicados, lo que se considera una de las limitantes de este estudio. Los artículos publicados en las revistas evaluadas, mostrados tanto en la tabla 1, indican una distribución de los porcentajes. 
Tabla 1

Artículos publicados por revista

\begin{tabular}{lcc}
\hline \multicolumn{1}{c}{ Título de la revista } & Cantidad & $\%$ \\
\hline Synthesis & 500 & 42,55 \\
Excelencia Administrativa & 246 & 20,93 \\
Lecturas Jurídicas & 221 & 18,80 \\
Tecnociencia & 77 & 6,55 \\
READICEA & 50 & 4,25 \\
DOXA & 47 & 4,00 \\
Metamorfosis & 34 & 2,89 \\
\hline Total & $\mathbf{1 , 1 7 5}$ & $\mathbf{1 0 0}$ \\
\hline
\end{tabular}

Los mismos artículos publicados, antes analizados por publicación, fueron agrupados por área de la ciencia según secciona el modelo propuesto por SNI, donde el área V (ciencias sociales) y IV (humanidades y ciencias de la conducta) agrupan el $41.44 \%$ y $40.25 \%$ respectivamente y son los porcentajes mayores de publicación del total de las revistas (tabla 2). El área I (físico-matemáticas y ciencias de la tierra) sólo cuenta con cinco artículos publicados $(.4255 \%)$ el cual es el menor índice de publicación en las revistas evaluadas.

Tabla 2

Artículos por área de conocimientos

\begin{tabular}{lcc}
\hline \multicolumn{1}{c}{ Área } & Frecuencia & $\mathbf{\%}$ \\
\hline V. Ciencias sociales & 487 & 41,44 \\
IV. Humanidades y ciencias de la conducta & 473 & 40,25 \\
III. Medicina y ciencias de la salud & 78 & 6,63 \\
VI. Biotecnología y ciencias agropecuarias & 67 & 5,70 \\
VII. Ingeniarías & 46 & 3,91 \\
II. Biología y química & 19 & 1,61 \\
I. Físico-matemáticas y ciencias de la tierra & 5 & 0,425 \\
\hline Total & $\mathbf{1 1 7 5}$ & $\mathbf{1 0 0}$ \\
\hline
\end{tabular}

La captura de artículos de cada revista fue evaluada además de acuerdo a los rangos de edades extremas de los años de publicación, tal como se indica en la tabla 3, en donde la de mayor rango correspondió a la revista Synthesis con 20 
años, seguida por Metamorfosis con 10 años y las de menor rango con de edades extremas con cuatro años (Tecnociencia y READICEA).

Tabla 3

Edades extremas de captura

\begin{tabular}{lcc}
\hline \multicolumn{1}{c}{ REVISTA } & AÑO MENOR & AÑ O MAYOR \\
\hline Synthesis & 1990 & 2010 \\
Excelencia Administrativa & 2004 & 2010 \\
Lecturas Jurídicas & 2006 & 2011 \\
Tecnociencia & 2007 & 2011 \\
READICEA & 2007 & 2011 \\
DOXA & 2005 & 2010 \\
Metamorfosis & 1998 & 2008 \\
\hline
\end{tabular}

Respecto al promedio de artículos por revista evaluada calculados con base en el universo de estudio son: Lecturas Jurídicas con 13.81\%, DOXA 11.75\%, Excelencia Administrativa 11.71\%, REDICEA $10.00 \%$, Metamorfosis $8.50 \%$, Synthesis $7.46 \%$ y Tecnociencia con $3.34 \%$.

Los artículos identificados dentro del universo de investigación, de acuerdo a la materia disciplinar en la que se ubican (siguiendo nuevamente el modelo clasificatorio del SNI), indican de acuerdo al anexo 1.

Respecto a los artículos que incluyen lista de referencias, se contabilizaron la totalidad del $57.70 \%$ que no contaban con ella y un $42.29 \%$ que sí contaban con referencias (Figura 1). Los artículos que fueron identificados con el indicador de NO incluyen referencias fue debido, como se menciona con anterioridad en el apartado de metodología, a que no se contó con el artículo fuente o por motivos de la misma naturaleza del documento, por lo cual, para estudios métricos de información con aplicación de leyes métricas no es factible su utilización ya que no arrojan resultados, por tanto, para acciones posteriores de esta investigación únicamente se tomará en cuenta el porcentaje de documentos identificados que cuentan con una lista de referencia formalmente insertada dentro del mismo. 
Figura 1

Artículos con o sin referencias

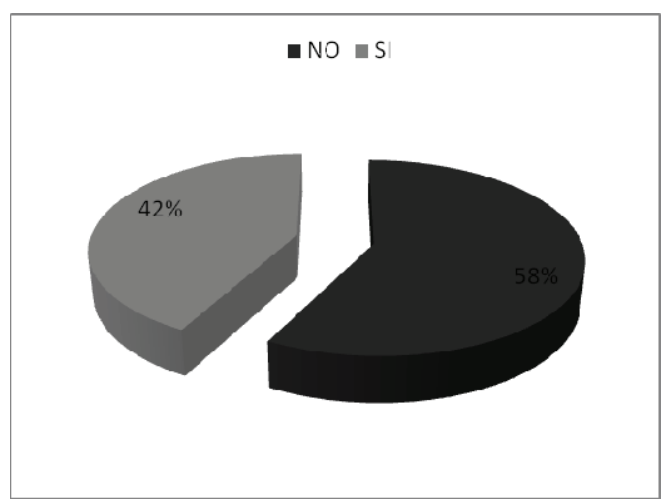

\subsection{APLICACIÓN DE LEYES MÉTRICAS}

A continuación se abordan los resultados obtenidos ante la aplicación de las leyes métricas consideradas para este estudio. Su estructura principal se compone de: autores, concentración-dispersión y obsolescencia, tanto por área temática como por tipo.

a) Autores. La población de autores encontrada en la muestra es de 1,122 sujetos distintos. Para el modelo del elitismo de Price en donde $\mathrm{n}=\sqrt{N}$ por lo que $\mathrm{n}=\sqrt{1122}, \mathrm{n}=33.4962$ (tabla 4), cuyo resultado representa la identificación de la élite de publicaciones explicada posteriormente.

Tabla 4

Publicación por grupos

\begin{tabular}{ccc}
\hline $\begin{array}{c}\text { Cantidad de } \\
\text { autores }\end{array}$ & Frecuencia & $\%$ \\
\hline Sin firma & 4 & 0,34 \\
1 & 726 & 61,78 \\
2 & 117 & 9,95 \\
3 & 181 & 15,40 \\
4 & 59 & 5,02 \\
5 & 40 & 3,40 \\
6 & 25 & 2,12 \\
7 & 12 & 1,02 \\
8 & 6 & 0,51
\end{tabular}




\begin{tabular}{ccc}
\hline $\begin{array}{c}\text { Cantidad de } \\
\text { autores }\end{array}$ & Frecuencia & \% \\
\hline 9 & 2 & 0,17 \\
10 & 1 & 0,08 \\
11 & 2 & 0,17 \\
\hline Total & $\mathbf{1 1 7 5}$ & $\mathbf{1 0 0}$ \\
\hline
\end{tabular}

A partir de los resultados arrojados en cuanto a publicación por grupos, se logró identificar la élite de publicación en revistas periféricas de la $\mathrm{UACH}$, en donde se observa una concentración en 11 autores (anexo 2), en su totalidad docentes de la propia institución, los cuales observan las siguientes regularidades: cinco poseen grado de doctor y seis de maestría; de acuerdo al área disciplinar: ocho pertenecen al área de contaduría y administración, dos a derecho y uno a química. Solamente uno de los autores tienen pertenencia al SNI con publicaciones en revistas arbitradas e indexadas, el resto centran su producción en revistas propias de la UACH.

Para el caso de la publicación por grupos, se tomaron en cuenta 1,171 artículos como el universo investigativo, donde los autores independientes firmantes son 726 que representa un $61.99 \%$ de la totalidad, 117 los que publican por parejas simboliza al 9.99\%, los grupos formales de tres autores son de 181 (un $15.45 \%$ de la totalidad), con cuatro autores firmantes son 59 artículos (5.03\%) y en grupos de cinco o más autores se cuentan 88 artículos acumulados (7.51\%), tales resultados son representados en la tabla 5 .

Tabla 5

Publicación por grupo (acumulado)

\begin{tabular}{lcc}
\hline Cantidad de autores & Frecuencia & $\mathbf{\%}$ \\
\hline 1 Autor & 726 & 61.99 \\
2 Autores & 117 & 9,99 \\
3 Autores & 181 & 15,45 \\
4 Autores & 59 & 5,03 \\
5 Autores o más & 88 & 7,51 \\
\hline Total & $\mathbf{1 1 7 1}$ & $\mathbf{1 0 0}$ \\
\hline
\end{tabular}

La aplicación de los modelos matemáticos en los resultados se representa por tablas debido a la gran cantidad de datos estructurados que de la aplicación de ellos resultan. Para el caso del modelo matemático de Lotka, el cual indica a los autores más productivos en los temas específicos (Gorbea Portal, 2005), para 
efectos de este estudio la manera en que está estructurado es de forma institucional no por temas, ya que como menciona Máltras Barba (2003), la suma de productos de sus centros o unidades forma un agregado con sentido que puede ayudar en la detección de diferencias entre las instituciones, en este caso está aplicado para encontrar los patrones internos de la institución.

La estimación generada para hacer el comparativo con el modelo matemático de Lotka, en donde 780 son autores con una mención (69.45\%), para dos autores corresponde a un cuarto de esta cantidad un (17.36\%) según lo estimado en el modelo, el porcentaje observado es $14.33 \%$; tres menciones es un noveno de una sola mención un $7.71 \%$ en el estimado y el observado es $5.07 \%$; cuatro menciones un dieciseisavo es $4.34 \%$ estimado y el observado corresponde $2.84 \%$. Los totales completos aparecen descritos en la tabla 6.

Tabla 6

Conteo de menciones

\begin{tabular}{ccccc}
\hline Menciones & Cuenta & $\mathbf{\%}$ & Estimación Lotka & $\begin{array}{c}\text { \% Estimación } \\
\text { Lotka }\end{array}$ \\
\hline 1 & 780 & $69,4568 \%$ & 780 & $69,4568 \%$ \\
2 & 161 & $14,3366 \%$ & 195 & $17,3642 \%$ \\
3 & 57 & $5,0757 \%$ & 87 & $7,7174 \%$ \\
4 & 32 & $2,8495 \%$ & 49 & $4,3411 \%$ \\
5 & 16 & $1,4248 \%$ & 31 & $2,7783 \%$ \\
6 & 14 & $1,2467 \%$ & 22 & $1,9294 \%$ \\
7 & 8 & $0,7124 \%$ & 16 & $1,4175 \%$ \\
8 & 9 & $0,8014 \%$ & 12 & $1,0853 \%$ \\
9 & 9 & $0,8014 \%$ & 10 & $0,8575 \%$ \\
10 & 15 & $1,3357 \%$ & 8 & $0,6946 \%$ \\
11 & 6 & $0,5343 \%$ & 6 & $0,5740 \%$ \\
12 & 4 & $0,3562 \%$ & 5 & $0,4823 \%$ \\
13 & 3 & $0,2671 \%$ & 5 & $0,4110 \%$ \\
16 & 1 & $0,0890 \%$ & 3 & $0,2713 \%$ \\
17 & 2 & $0,1781 \%$ & 3 & $0,2403 \%$ \\
18 & 1 & $0,0890 \%$ & 2 & $0,2144 \%$ \\
20 & 1 & $0,0890 \%$ & 2 & $0,1736 \%$ \\
21 & 1 & $0,0890 \%$ & 2 & $0,1575 \%$ \\
30 & 1 & $0,0890 \%$ & 1 & $0,0772 \%$ \\
54 & 1 & $0,0890 \%$ & 0 & $0,0238 \%$ \\
\hline Total & 1123 & & & \\
\hline
\end{tabular}


Con respecto al índice de coautoría, este es calculado por la cantidad de autores firmantes entre la cantidad de documentos. Por lo que el índice de coautoría calculado es de 1.86. El grado de colaboración o la tasa promedio de documentos coautorados está indicado por la suma de documentos donde están firmados por más de un autor sobre la cantidad total de documentos, lo que resulta con un .38 (resultados finales presentados en la tabla 7).

Tabla 7

Índice de coautoría

\begin{tabular}{lll}
\hline Índice de coautoría & 1,86 & $\begin{array}{l}\text { Autores firmantes por } \\
\text { documentos }\end{array}$ \\
\hline $\begin{array}{l}\text { Tasa promedio de } \\
\text { documentos coautorados o } \\
\text { grado de colaboración }\end{array}$ & 0,38 & $\begin{array}{l}\text { Tasa documentos con } \\
\text { autoría múltiple }\end{array}$ \\
\hline
\end{tabular}

b) Concentración-Dispersión temática. Para la posterior aplicación de los modelos métricos, como es el caso del modelo matemático de Bradford, el cual determina el número de revistas productivas por temas, se realiza una matriz que divide los artículos en áreas de conocimiento contra las revistas que lo publican (tablas 8 y 9), observándose que en los casos de la mayoría de las áreas no hay una publicación constante de todas las revistas para aplicar un estudio métrico bajo el modelo matemático aquí abordado, ya que la mayor concentración corresponde a las áreas IV. Humanidades y ciencias de la conducta y V. Ciencias sociales, siendo éstas de la que se desarrolla el análisis de datos posterior.

Tabla 8

Matriz de artículos revistalárea

\begin{tabular}{ccccccccc}
\hline \multirow{2}{*}{ Nombre de la revista } & \multicolumn{7}{c}{ Áreas de conocimiento } \\
\cline { 2 - 9 } & I & II & III & IV & V & VI & VII & Totales \\
\hline DOXA & & & & 15 & 32 & & & 47 \\
Excelencia Administrativa & & 1 & 1 & 106 & 135 & & 3 & 246 \\
Lecturas Jurídicas & & 3 & & 4 & 213 & & 1 & 221 \\
Metamorfosis & & & & 33 & 1 & & & 34 \\
READICEA & & & & 34 & 16 & & & 50 \\
Synthesis & 1 & 9 & 67 & 277 & 86 & 33 & 27 & 500 \\
Tecnociencia & 4 & 6 & 10 & 4 & 4 & 34 & 15 & 77 \\
\hline Totales & 5 & 19 & 78 & 473 & 487 & 67 & 46 & 1175 \\
\hline
\end{tabular}

(Área I. Físico-matemáticas y ciencias de la tierra; Área II. Biología y química; Área III. Medicina y ciencias de la salud; Área IV. Humanidades y ciencias de la conducta; Área V. Ciencias sociales; Área VI. Biotecnología y ciencias agropecuarias; Área VII. Ingenierías) 
Según los resultados obtenidos se observa que la revista que incluye la primer zona es la revista Synthesis, las revistas de la segunda zona son Excelencia Administrativa y Lecturas Jurídicas y la tercer zona incluye el restante de las revistas: Tecnociencia, REDICEA, DOXA y Metamorfosis. A partir de la división de zonas se obtienen los resultados de $m$ la cual representa la cantidad de revistas que producen en la zona $1, p=$ a la producción de las revistas y $r=$ al promedio por publicaciones periódicas de la zona. De la misma manera están los indicadores de zona 2 , son $m l, p_{1}$ y $r_{1}$ y $m_{2}, p_{2}$ y $r_{2}$ para la zona número 3 . Los resultados son los siguientes: en la zona $1 \mathrm{p}=1, \mathrm{~m}=500$ y $\mathrm{r}=500$; en la zona $2, \mathrm{p}_{1}=2, \mathrm{~m}_{1}=467$ y $\mathrm{r}_{1}=233.5$ y para la zona $3, \mathrm{p}_{2}=4, \mathrm{~m}_{2}=208$ y $\mathrm{r}_{2}=52$, lo que nos indica que la revista de Synthesis ocupa por completo la zona de mayor producción que es la 1.

En relación con los artículos publicados en las revistas del área de humanidades existen un total de 473 artículos de los que se dividen la publicación de la siguiente manera: Synthesis con 277, Excelencia Administrativa con 106, REDICEA con 34, Metamorfosis con 33, DOXA con 15, Lecturas Jurídicas con 4 y Tecnociencia con 4 artículos (Tabla 20). Los valores obtenidos en las zonas son: $\mathrm{p}=1, \mathrm{~m}=277, \mathrm{r}=277, \mathrm{p}_{1}=3, \mathrm{~m}_{1}=173, \mathrm{r}_{1}=57.67, \mathrm{p}_{2}=3, \mathrm{~m}_{2}=23$ y $\mathrm{r}_{2}=7.67$.

Para las revistas de ciencias sociales (Área V) correspondieron 487 artículos, donde la revista Lecturas Jurídicas observan una publicación de 213, Excelencia Administrativa con 135, Synthesis con 86, DOXA con 32, REDICEA con 16, Tecnociencia con 4 y Metamorfosis con 1 . Los valores para las zonas son: $\mathrm{p}=1$, $\mathrm{m}=213, \mathrm{r}=213, \mathrm{p}_{1}=1, \mathrm{~m}_{1}=135, \mathrm{r}_{1}=135, \mathrm{p}_{2}=5, \mathrm{~m}_{2}=139 \mathrm{y} \mathrm{r}_{2}=27.80$.

Tabla 9

Zonas en el modelo matemático de Bradford por revista

\begin{tabular}{ccccc}
\hline A & B & $\mathrm{C}=(\mathrm{A} X \mathrm{~B})$ & $\mathrm{D}$ & $\mathrm{E}$ \\
\hline \multirow{2}{*}{ Títulos revistas } & Artículos revistas & Total de artículos & $\begin{array}{c}\text { Acumulado } \\
\text { revistas } \\
N\end{array}$ & $\begin{array}{c}\text { Acumulado } \\
\text { artículos } \\
R(n)\end{array}$ \\
\hline 1 & & & $N$ & 500 \\
1 & 500 & 500 & 1 & 746 \\
1 & 246 & 246 & 2 & 967 \\
1 & 221 & 221 & 3 & 1044 \\
1 & 77 & 77 & 4 & 1094 \\
1 & 50 & 50 & 5 & 1141 \\
1 & 47 & 47 & 6 & 1175 \\
\hline
\end{tabular}

Para los efectos prácticos de la aplicación de este estudio métrico de información, solamente con la detección de las zonas I de concentración es suficiente, en el caso del área IV es la revista Synthesis y en el área V es Lecturas Jurídicas, las revistas con una mayor concentración en su respectiva área temática. 
Los números restantes de las tablas como las columnas de producción calculada como las diferencias entre otros, se mantienen en las tablas originales para posteriores investigaciones.

c) Modelo de elitismo de Price. Toda la población de tamaño $\boldsymbol{N}$ tiene una élite que va a ser igual a su raíz cuadrada. La denominación de esta elite puede apuntar a un núcleo selecto de la población total y su formulación matemática puede ser: $\mathrm{n}=\mathrm{N}^{1 / 2}$, donde $\mathrm{N}=$ a la población total, $\mathrm{n}=\mathrm{a}$ la raíz cuadrada de la población, es decir, la élite o núcleo.

Los tipos de autores que considera este modelo son dos: personales y corporativos. Evalúa los indicadores de autoría: índice de coautoría y tasa de documentos coautorados. El índice de coautoría equivale a $\mathrm{Ic}=\mathrm{af} / \mathrm{d}$, donde: af $=$ cantidad de autores firmantes, $\mathrm{d}=$ cantidad de documentos y la tasa de documentos coautorados. $\mathrm{Tdc}=\mathrm{Cm} / \mathrm{d}$, donde: $\mathrm{Cm}=$ Cantidad de documentos con autoría múltiple.

De acuerdo al índice de obsolescencia de Price, el cálculo del indicador de la obsolescencia puede obtenerse a partir de las referencias que sustentan al documento. Por tanto, los resultados de la obsolescencia se encuentran representados de dos maneras, la primera es por revista (lo cual indica el grado de obsolescencia institucional ya que las revistas por sí mismas representan a un sector de la propia institución general) y la segunda representación de resultados es por área temática.

El valor numérico del índice de Price asume para la literatura de archivo entre los límites:

a) $\mathrm{Ip}=\mathrm{Ro} / \mathrm{Rt}$; donde $\mathrm{Ro}=\mathrm{Referencias}$ operativas y $\mathrm{Rt}=\mathrm{Referencias}$ Totales

b) Referencias operativas: $<5$ años

c) Referencias de archivo: $>5$ años

d) Con respecto a la fecha de publicación del documento.

e) $22 \%$ crecimiento normal

f) $39 \%$ crecimiento rápido

g) Para la literatura de efecto operativo: $75 \%$ al $80 \%$

h) Promedio para todas las ciencias: $50 \%$

El modelo de elitismo de Price fue evaluado desde dos perspectivas: por revista evaluada (anexo 3) y por área de conocimiento (anexo 4) y en cada caso se abordaron los aspectos de obsolescencia, vida media y tasa de envejecimiento promedio del total de las revistas participantes en el estudio. Para el caso específico de la obsolescencia por área temática, como se ha indicado a través del documento, el modelo de clasificación temática por área de conocimiento utilizado en esta investigación es el propuesto por el SNI para identificar disciplinas científicas. 


\section{DISCUSIÓN}

Se evaluaron siete revistas editadas en diversas entidades administrativas y académicas de la UACH, de las cuales se observa, que las revistas DOXA y Metamorfosis son las que cuentan con más irregularidad en su publicación. En cambio, las restantes (Synthesis, Excelencia Administrativa, Lecturas Jurídicas, Tecnociencia y REDICEA) se encuentran en una publicación constante, lo cual permite regular sus estándares de evaluación métrica correctamente, lo que podría permitir a futuro el cambio de estatus en su condición de evaluación (de divulgación a científica).

Las publicaciones evaluadas observan una regulación básica en la selección de artículos a publicar, considerando que sus comités editoriales no observen la exigencia requerida por las revistas de alta calidad. Esto provoca la aceptación de todas las propuestas de artículos recibidos, lo que se proyecta en distintas irregularidades editoriales, tales como: problemas de redacción, ortografía y sintaxis, además de que en ocasiones los artículos no incluyen las referencias completas y sin un formato normalizado de integración de sus datos bibliográficos y de manuales de estilo precisos.

Cuatro publicaciones evaluadas están enfocadas en un área científica disciplinar particular (Lecturas Jurídicas, Excelencia Administrativa, REDICEA y DOXA); una más (Metamorfosis), observa una inconstancia visible en su periodicidad de publicación, y aunque pudiera considerarse especializada en una disciplina particular, el exceso de programas profesionales que se ofertan en la entidad que la edita, la convierte en multidisciplinaria dentro del área de las humanidades. Las dos publicaciones restantes del universo evaluado (Tecnociencia y Synthesis) observan ser multidisciplinaria, sin embargo son las revistas que cuentan con un comité editorial más preciso, con mayor regularidad en su publicación, además de considerarse como las más viables a cambiar de estatus respecto a los criterios de evaluación de la calidad.

Dado el comportamiento en las revistas evaluadas, respecto al origen de las publicaciones, las cuales provienen en su mayoría de los mismos docentes de la $\mathrm{UACH}$, existe una tendencia fuerte a identificar autores oportunistas, tanto en autores tendientes a escribir en disciplinas distintas a la propia área de estudio (una inmensa mayoría desarrolla contribuciones del área de educación por encima de su propia disciplina), como al exceso de coautoría sin una participación real de todos los autores citados en cada artículo.

Por tanto, la identificación de élites intelectuales se basa en relación con el análisis de los resultados obtenidos de la delimitación de este estudio, sin embargo, no garantiza que los autores que aparecen como oportunistas en este estudio observen o no índices adecuados de comunicación científica hacia el exterior de la propia institución, y viceversa, algunos autores que aparecen con 
alto grado de productividad científica en publicaciones internas carezcan una trayectoria externa que los posicione en otros ámbitos de la ciencia.

Considerando las condiciones generales de las publicaciones internas de la UACH con base en su análisis bibliométrico, se hace necesario:

a) Establecer el compromiso por parte de las entidades que las editan a mantener una constancia y mejora en la recepción y evaluación de los artículos a publicar, así como en la regularidad de la publicación

b) La elección de comités científicos de evaluación con reconocido prestigio y en la especialidad

c) Definición de políticas editoriales institucionales para la creación de nuevas publicaciones y mejorar la estandarización de las actuales

d) Aumentar la visibilidad de las publicaciones (la cual es relativamente nula o muy baja), provocando un aumento en el nivel de calidad, pasando de la mera divulgación al estatus de revista científica (arbitrada e indizada).

Además, como resultado de esta investigación, se espera la posibilidad de otros estudios vinculados a la generación de un modelo formal de evaluación de la producción y comunicación científica, cuya regularidad cubra la expectativa institucional y se convierta en un observatorio científico, que incluso vincule las publicaciones internas con el resto de la producción científica de los docentes e investigadores de la $\mathrm{UACH}$.

Como recomendación adicional para posteriores investigaciones de esta índole, es necesario realizar estudios métricos de la información que permitan calcular el nivel de impacto de los artículos publicados por las revistas periféricas con mayor regularidad en sus ediciones, contando así con un respaldo para solicitudes de cambio de estatus, anexionando las revistas en un nivel superior de calidad y probable ingreso al grupo de publicaciones de corriente principal o mainstream, garantizando así la visibilidad de la información científica contenida.

\section{CONCLUSIONES}

Las revistas periféricas deberán experimentar una clasificación más allá de la mera no inclusión dentro del grupo de corriente principal o mainstream, considerando para ello aspectos precisos de calidad editorial. Se considera a aquellas publicaciones periféricas de alta calidad como representantes de un medio idóneo para la divulgación de la ciencia gestada por académicos universitarios.

El desarrollo rutinario de estudios bibliométricos puede propiciar la visualización de indicadores internos de calidad en los contenidos de las revistas periféricas, pero difícilmente se lograran identificar elementos de impacto y visibilidad en una dimensión extrínseca de los documentos publicados.

El caso particular estudiado, que caracteriza la edición de revistas periféricas de generación interna en donde se muestra libertad disciplinar de las propuestas publicadas, dificultad de identificación de elites intelectuales, 
multidisciplinariedad de las publicaciones y carencia de rigor editorial de fondo y forma, puede ser modelo común de las universidades mexicanas (especialmente públicas), sin embargo, no es generalizable, ya que se tiene referencia de instituciones de educación superior locales y extranjeras que contribuyen a la divulgación de la ciencia a través de medios formales y sistemáticos.

Aunque esta investigación se limita a una sola entidad educativa, es importante indicar la posibilidad de desarrollar estudios similares en otras universidades públicas estatales en México, lo cual permitiría conocer las condiciones generales que imperan en cuestiones editoriales, ya que se infiere que el fenómeno se repite, al observar un bajo número de publicaciones periódicas incluidas dentro de los catálogos nacionales e internacionales de revistas de corriente principal o mainstream, regularmente generadas por organismos que ostentan el nivel de universidades de carácter nacional.

\section{LISTA DE REFERENCIAS}

AMERICAN LIBRARY ASSOCIATION. [En línea], 2011. Disponible en: $\mathrm{http}: / /$ www.ala.org. [Consulta: 1 de junio, 2012]

DÁVILA RODRÍGUEZ, M. y otros. "Bibliometría: conceptos y utilidad para el estudio médico y la formación profesional". Salud Uninorte, 2009, 25/2: 319330.

GORBEA PORTAL, S. Modelo teórico para el estudio métrico de la información documental. México: Ediciones TREA, 2005.

GORBEA PORTAL, S., ÁVILA URIZA, M. "Publicaciones seriadas en ciencias bibliotecológicas y de la información: su estado actual". Investigación Bibliotecológica, 2009, agosto, 23/48: 179-209.

GORBEA PORTAL, S., SUÁREZ-BALSEIRO, C. A. "Análisis de la influencia y el impacto entre revistas periféricas no incluidas en el Science Citation Index". Revista Interamericana de Bibliotecología, 2007, julio-diciembre, 30/2: 47-70.

GUTIÉRREZ COUTO, U., BLANCO PÉREZ, A., CASAL ACCIÓN, B. (2004, diciembre). "Cómo realizar una comunicación científica: estructura de la comunicación científica". Revista Gallega de Terapia Ocupacional TOG, 2004, diciembre, 1.

Jiménez Sánchez. J., Castañeda Hernández, M.A. “Algunas consideraciones sobre la evaluación de la calidad de las revistas". Rev Enferm IMSS, 2003, 11/1: 1-3.

INTERNATIONAL COUNCIL FOR SCIENCE. Guidelines for printing and publishing. Paris, Francia: ICSU Press, 1999.

LÓPEZ-LÓPEZ, W. "De la cienciometría y los procesos de valoración de la producción intelectual". Universitas Psychologica, 2009, mayo-agosto, 8/2.

LÓPEZ ORNELAS, M. Diseño y validación de un instrumento para evaluar revistas académicas electrónicas en internet. Tesis (Maestría en Ciencias Educativas), Universidad de Baja California, 2004 
LÓPEZ ORNELAS, M., CORDERO ARROYO, G. "Un intento por definir las características generales de las revistas académicas electrónicas". Razón y Palabra, 2005, 43, febrero-marzo.

MALTRÁS BARBA, B. Los indicadores bibliométricos: Fundamentos y aplicación al análisis de la ciencia. España: Ediciones Trea, 2003.

Márquez Nerey, E. Percepción social de la ciencia de un grupo de adolescentes de la ciudad de México. Tesis (Doctor en Psicología, Universidad Nacional Autónoma de México, 2010).

MENDOZA, S., PARAVIC, T. (2006, junio). "Origen, clasificación y desafíos de las revistas científicas”. Investigación y Posgrado, 2006, 21/1, junio.

NÚÑEZ JOVER, J. La ciencia y la tecnología como procesos sociales: lo que la educación cientifica no debería de olvidar. La Habana, Cuba: Félix Varela, 2006.

RÍOS ORTEGA, J. "Evaluación de normalización de las revistas contenidas en el Índice del Consejo Nacional de Ciencia y Tecnología". Investigación Bibliotecológica, 2006, enero-junio, 20/40: 121-148.

SÁNCHEZ HERNÁNDEZ, A., DELGADO SÁNCHEZ, A., SORIA RAMÍREZ, V. "Las revistas científicas periféricas en un entorno cambiante: el reto de las revistas científicas periféricas latinoamericanas". IBERSID, 2007: 331-342.

SAMAR, M.E., ÁVILA, R.E. Guía para la elaboración y publicación de artículoscientíficos y la recuperación de la información en ciencias de la salud. Córdoba, Argentina:Universidad Nacional de Córdoba (UNC), 2004.

SATIÉN QUEZADA, E., GORBEA PORTAL, S. "De la bibliotecología al sistema de conocimientos científicos bibliológico-informativo". Investigación Bibliotecológica, 1994, enero-junio, 8/16.

SISTEMA NACIONAL DE INVESTIGADORES. [En línea], 2012. Disponible en: www.conacyt.gob.mx/SNI/Index_SNI.html. [Consulta: 1 de junio, 2012]

UNESCO, Oficina Regional de Ciencia para América Latina y el Caribe. Reunión de Especialistas en Información Científica Digital. Montevideo: UNESCO, 2003.

Anexo 1. Total de artículos por materia

\begin{tabular}{lcc}
\hline \multicolumn{1}{c}{ Ciencia } & Frecuencia & \% \\
\hline Educación & 207 & 17,61 \\
Derecho y jurisprudencia & 168 & 14,29 \\
Administración & 153 & 13,02 \\
Literatura & 112 & 9,53 \\
Ciencia política y administración pública & 101 & 8,59 \\
Filosofía & 76 & 6,46 \\
Medicina & 51 & 4,34 \\
Agronomía & 50 & 4,25 \\
Ambiental & 37 & 3,14
\end{tabular}




\begin{tabular}{|c|c|c|}
\hline Ciencia & Frecuencia & $\%$ \\
\hline Bellas artes & 31 & 2,63 \\
\hline Economía & 23 & 1,95 \\
\hline Comunicación & 20 & 1,70 \\
\hline Historia & 15 & 1,27 \\
\hline Sociología y prospectiva & 15 & 1,27 \\
\hline Zootecnia & 14 & 1,19 \\
\hline Ciencias de la salud & 13 & 1,10 \\
\hline Antropología & 12 & 1,02 \\
\hline Nutrición & 12 & 1,02 \\
\hline Biología & 11 & 0,93 \\
\hline Psicología & 8 & 0,68 \\
\hline Química & 8 & 0,68 \\
\hline Lingüística & 6 & 0,51 \\
\hline Contabilidad & 5 & 0,42 \\
\hline Ciencias de materiales & 4 & 0,34 \\
\hline Biblioteconomía & 3 & 0,25 \\
\hline Biotecnología & 3 & 0,25 \\
\hline Computación & 3 & 0,25 \\
\hline Industrial & 3 & 0,25 \\
\hline Civil & 2 & 0,17 \\
\hline Geografía & 2 & 0,17 \\
\hline Arquitectura & 1 & 0,08 \\
\hline Educación física & 1 & 0,08 \\
\hline Geografía humana & 1 & 0,08 \\
\hline Geología & 1 & 0,08 \\
\hline Odontología & 1 & 0,08 \\
\hline Pesca & 1 & 0,08 \\
\hline Textil & 1 & 0,08 \\
\hline Total & 1175 & $100 \%$ \\
\hline
\end{tabular}


Anexo 2. Élite de publicación en revistas periféricas de la UACH

\begin{tabular}{|c|c|c|}
\hline & Autores & Frecuencia \\
\hline \multirow{11}{*}{ 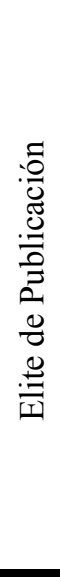 } & García Bencomo, Myrna Isela & 54 \\
\hline & Reyes López, Gerardo & 30 \\
\hline & Ávila Wall, Olga Leticia & 21 \\
\hline & Arroyo Ávila, René & 20 \\
\hline & Gallegos Cereceres, Víctor Manuel & 18 \\
\hline & Cobos Campos, Amalia Patricia & 17 \\
\hline & Torres Medina, Rubén & 17 \\
\hline & Hurtado Licón, Xavier & 16 \\
\hline & Martínez Ramos, Pedro & 13 \\
\hline & Ollivier Fierro, Juan Oscar & 13 \\
\hline & Velázquez Pérez, Francisco Cervando & 13 \\
\hline & González Terrazas, Armando & 12 \\
\hline & Ponce García, Bertha Alicia & 12 \\
\hline & Sánchez, Luis Raúl & 12 \\
\hline & Sapién Aguilar, Alma Lilia & 12 \\
\hline & Araiza Zapata, Patricia Aracely & 11 \\
\hline & González González, Manuel Benjamín & 11 \\
\hline & Gutiérrez Diez, María Del Carmen & 11 \\
\hline & Hernández Rodríguez, Ofelia Adriana & 11 \\
\hline & Pérez Chávez, Teresa De Jesús & 11 \\
\hline & Treviño Miramontes, Yolanda Esperanza & 11 \\
\hline & Álvarez Terrazas, José Alfonso & 10 \\
\hline & Aleláis, Jesús & 10 \\
\hline & Arras Vota, Ana María de Guadalupe & 10 \\
\hline & Caraveo Valdez, Jaime A. & 10 \\
\hline & Carrete Meza, Octavio & 10 \\
\hline & Escobedo Cisneros, Hilda Cecilia & 10 \\
\hline & García Quintana, Raymundo & 10 \\
\hline & Martínez Murillo, Elva Norma & 10 \\
\hline & Ojeda Barrios, Dámaris Leopoldina & 10 \\
\hline & Paredes Aguirre, Manuel Alberto & 10 \\
\hline & Piñón Howlet, Laura Cristina & 10 \\
\hline
\end{tabular}


Javier Tarango, Arturo Iván Ruiz-Domínguez $\quad$ Revistas periféricas de generación interna en la...

\begin{tabular}{lc}
\hline \multicolumn{1}{c}{ Autores } & Frecuencia \\
\hline Polanco Rodríguez, Irma & 10 \\
Ramírez Lujan, Heriberto & 10 \\
Robles Villa, Jesús & 10 \\
Torres Medina, Rodolfo & 10 \\
\hline 9 Docentes & 9 \\
8 Docentes & 9 \\
7 Docentes & 8 \\
6 Docentes & 14 \\
5 Docentes & 16 \\
4 Docentes & 32 \\
3 Docentes & 57 \\
2 Docentes & 161 \\
1 Docente & 780 \\
\hline Total & $\mathbf{2 , 2 7 0}$ \\
\hline
\end{tabular}

